Eur J Clin Chem Clin Biochem

1995; 33:859-864

(ㄷ) 1995 Walter de Gruyter \& Co.

Berlin · New York

\title{
Usefulness of Reference Materials in Calibration of Enzyme Activities ${ }^{1}$ )
}

\author{
By Jean-Marc Lessinger ${ }^{1}$, Georges Férard ${ }^{1}$, Denis Grafmeyer ${ }^{2}$, Dominique Labbé ${ }^{3}$, Irène Maire ${ }^{4}$, \\ Françoise Schiele ${ }^{5}$ and Anne Vassault ${ }^{3}$ \\ ${ }^{1}$ Laboratoire de Biochimie Appliquée et Laboratoire du Centre de Traumatologie et d'Orthopédie, \\ Université Louis Pasteur de Strasbourg, Illkirch, France \\ 2 Laboratoire de Biochimie, Hôpital de la Croix-Rousse, Lyon, France \\ 3 Laboratoire de Biochimie A, Hôpital Necker-Enfants Malades, Paris, France \\ ${ }^{4}$ Laboratoire de Biochimie Pédiatrique, Hôpital Debrousse, Lyon, France \\ 5 Laboratoire du Centre de Médecine Préventive, Vandœuvre-lès-Nancy, France
}

(Received February 22/July 12, 1995)

Summary: $\alpha$-Amylase, alkaline phosphatase and $\gamma$-glutamyltransferase were studied in a multicentre evaluation. Analyses were performed on different patient samples. Each enzyme was assayed in two different laboratories at both 30 and $37^{\circ} \mathrm{C}$, with widely used reagent kits and with the IFCC reference method (if in existence). Results differed considerably according to the measurement procedure. Data also showed that it was not possible to employ a constant conversion factor for one enzyme and different techniques between 30 and $37^{\circ} \mathrm{C}$.

Calibration with three reference materials extensively improved the intermethod consistency for most of the tested measurement procedures. It was possible to transfer accuracy from the method used for the certification of the reference material to routine procedures, by using the reference material as calibrator. Temperature did not seem to be a crucial variable for the implementation of the enzyme calibrator approach.

\section{Introduction}

The method-dependency of the results of enzymic activity determinations presents problems of interpretation for clinicians. The aims of the present study were to evaluate:

- the degree of method-dependency of results obtained using routine conditions

- the effect of the use of appropriate enzyme calibrators on the improvement of between-method coherency.

This preliminary study was limited to $\alpha$-amylase (EC 3.2.1.1), alkaline phosphatase (EC 3.1.3.1), and $\gamma$-gluta-

1) Present study was performed by the working group "Matériaux de Référence en Enzymologie Clinique" (Responsible: J. M. Lessinger) from the section "Assurance de Qualite" (Coordinator: G. Férard) of Société Française de Biologie Clinique (SFBC). Members of the group: G. Férard, J. Frei, D. Grafmeyer, D. Labbé, J. M. Lessinger, I. Marie, M. Mathieu, F. Schiele, A. Vassault, A. Vialle. myltransferase (EC 2.3.2.2). $\alpha$-Amylase was chosen because of the diversity of used substrates. Alkaline phosphatase and $\gamma$-glutamyltransferase were chosen for the differences in the nature and concentration of buffer and substrate. Furthermore, reference methods $(1,2)$ and certified reference materials (CRM) are available for alkaline phosphatase and $\gamma$-glutamyltransferase $(3,4)$ and are being developed for $\alpha$-amylase.

\section{Materials and Methods}

General aspects

Five laboratories participated in this study. Each enzyme activity was determined independently in two laboratories, using automated procedures with the same reagent kits, at 30 and $37^{\circ} \mathrm{C}$, under routine conditions according to the procedure described by the manufacturer for each instrument. Each laboratory analysed specimens from its own patients (35 to 100 fresh sera, assayed in 6 to 10 series). Haemolysed, turbid or highly icteric samples were discarded. For each enzyme, samples with catalytic activity concentra- 
tions below the uppcr linearity limit of the methods were selected, taking care to ensure a homogeneous"distribution of the values in the range tested. In each run, the corresponding CRM was also assayed (tab. 1). Two CRM (CRM 319 and CRM 371 for $\gamma$-glutamyltransferase and alkaline phosphatase, respectively) were purchased from the Community Bureau of Reference (BCR), Brussels. The $\alpha$-amylase candidate reference material (CRM 318) prepared under the BCR auspices was provided by Prof. $F . J$. Gella (Barcelona).

\section{Reagents}

Various commercial kits of reagents were chosen because of their frequent use in Europe.

\section{a-Amylase catalytic activity concentration}

$\alpha$-Amylase was assayed with a kit containing maltotetraose (No. 63112, bioMérieux SA, Marcy-l'Étoile, France) and with kits containing different 4-nitrophenyloligosaccharides, i. e. 4-nitrophenylmalto- $\alpha$-heptaoside (No. 568589, Boehringer-Mannheim GmbH, Mannheim, Germany), ethylidene-4-nitrophenylmalto- $\alpha$-heptaoside (No. $1054671 \&$ 1054643, Boehringer-Mannheim), benzylidene-4-nitrophenylmalto- $\beta$-heptaoside (No. 63122, bioMérieux), 2chloro-4-nitrophenylmalto- $\alpha$-trioside (No. 63123 , bioMérieux) and 2-chloro-4-nitrophenylmalto- $\beta$-heptaoside (No. 12121, Merck, Darmstadt, Germany).

\section{Alkaline phosphatase catalytic activity concentration}

Alkaline phosphatase was determined using 4-nitrophenylphosphatate as substrate and two different buffer solutions. The first was diethanolamine in the kits from bioMérieux (No. 63609) and Boehringer-Mannheim (No. 415278) according to the DGKC recommended procedure. The second was 2-amino-2-methyl-1-propanol in the kits from bioMérieux (No. 63657), Biotrol (No. 03001, Biotrol, Paris, France), Boehringer-Mannheim (No. 396460) and Roche (No. 2813, Hoffmann-La Roche Ltd, Basle, Switzerland) according to the SFBC recommended procedure. Roche reagent in accordance with the IFCC recommendations (No. 0710571) was also selected in this study.

\section{$\gamma$-Glutamyltransferase catalytic activity concentration}

$\gamma$-Glutamyltransferase was assayed with Boehringer-Mannheim and Merck kits containing $\gamma$ - $L$-glutamyl-3-carboxy-4-nitroanilide (No. 158208 and No. 12191, respectively) and with bioMérieux and Biotrol kits containing $\gamma$ - $L$-glutamyl-4-nitroanilide as substrate (No. 63711 and No. A03027, respectively).

IFCC reference methods were also performed for alkaline phosphatase and $\gamma$-glutamyltransferase using home made reagents.

\section{Instruments}

$\alpha$-Amylase was run with a Cobas Mira analyser and a Cobas Fara analyser (Roche) in Laboratory 1 and Laboratory 2, respectively. Alkaline phosphatase was run with a Cobas Fara in Laboratory 2 and a Monarch apparatus (Instrumentation Laboratory, Milano, Italy) in Laboratory 3. $\gamma$-Glutamyltransferase was assayed with a Specific analyser (Kone Instrument Corporation, Espoo, Finland) in Laboratory 4 and a Cobas Mira in Laboratory 5.

For each enzyme and each method, instruments were set up according to the manufacturers' instructions. Thus, catalytic activity concentrations were first calculated using the factor based on the molecular absorption coefficient. Data were then recalculated using the corresponding CRM as calibrator.

\section{Data analysis}

The mean of all results obtained for patient samples was calculated independently in each laboratory before and after calibration by the selected CRM. Certified values established (or currently determined) by the BCR were used for the calibration of each measurement procedure: $254 \mathrm{U} / 1$ for alkaline phosphatase using the IFCC method at $30^{\circ} \mathrm{C}(1), 86.7 \mathrm{U} / \mathrm{h}$ for $\gamma$-glutamyltransferase using the IFCC method at $30^{\circ} \mathrm{C} \mathrm{(2)} \mathrm{and} 549 \mathrm{U} / 1$ for $\alpha$-amylase using 2chloro-4-nitrophenylmaltotrioside as substrate at $37^{\circ} \mathrm{C}$ under the following conditions: 2-chloro-4-nitrophenylmaltotrioside, 2.25 $\mathrm{mmol} / \mathrm{l}$; potassium thiocyanate, $900 \mathrm{mmol} / \mathrm{l}$; sodium chloride, 300 $\mathrm{mmol} / \mathrm{l}$; calcium chloride, $5 \mathrm{mmol} / \mathrm{l}$; 2-morpholino-ethanesulphonic acid, $50 \mathrm{mmol} / \mathrm{pH}\left(37^{\circ} \mathrm{C}\right) 6.28$.

\section{Results}

For each of the three enzymes assayed, results calculated with a theoretical calculation factor varied greatly according to the reagent used and temperature of measurement (figs. 1-3). Between-method dependency before calibration was more pronounced for $\alpha$-amylase and alkaline phosphatase than for $\gamma$-glutamyltransferase. Fig-

Tab. 1 Some characteristics of the enzyme reference materials used for calibration.

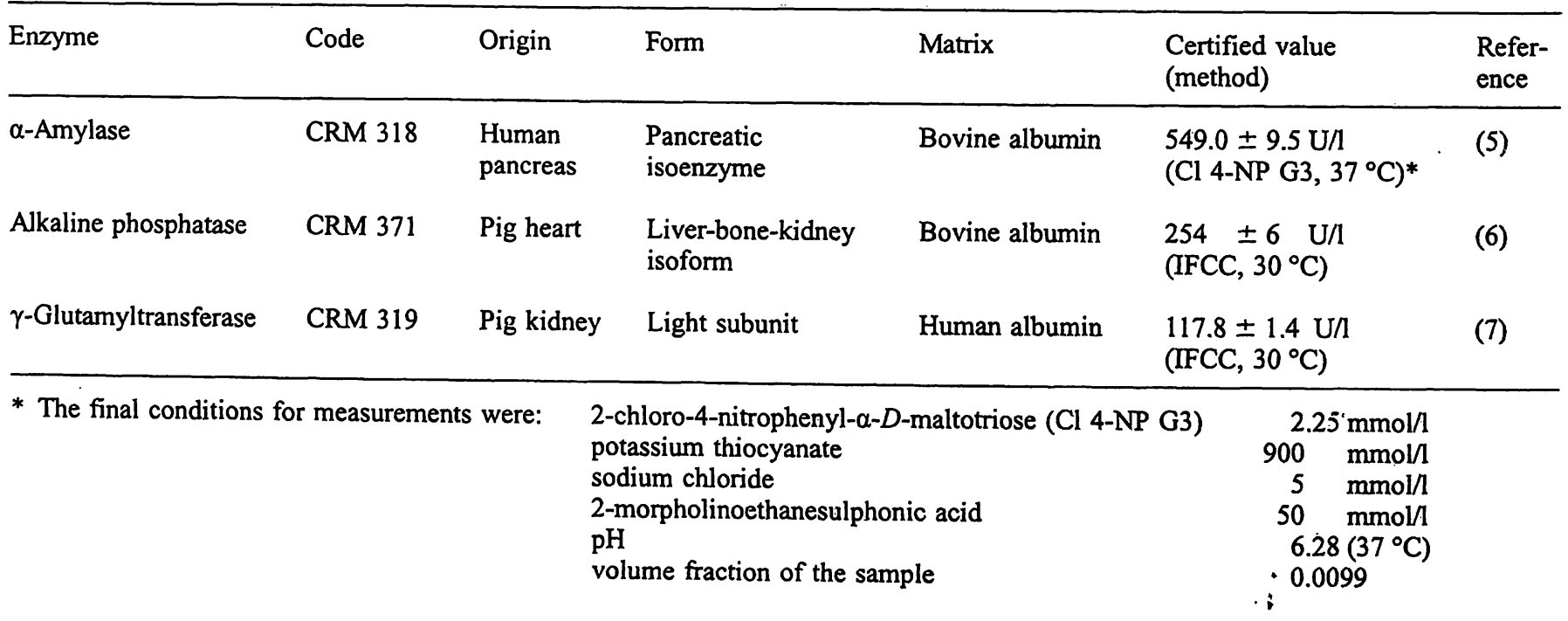


ures 1 to 3 indicate that the general patterns were similar in the two laboratories that assayed the same enzyme activity. Agreement between results in each participating laboratory was clearly improved by calibration. Nevertheless, the $\alpha$-amylase results obtained with maltotetraose after calibration still differ from those obtained with the other substrates employed in this study. This discrepancy was observed at both temperatures and in the two laboratories. For alkaline phosphatase, intermethod differences were not noticed. In contrast, after calibration, an intermethod disagreement was noted in the two laboratories for $\gamma$-glutamyltransferase results obtained with a kit containing a non-carboxylated substrate, compared with the results obtained with the other kits. Table

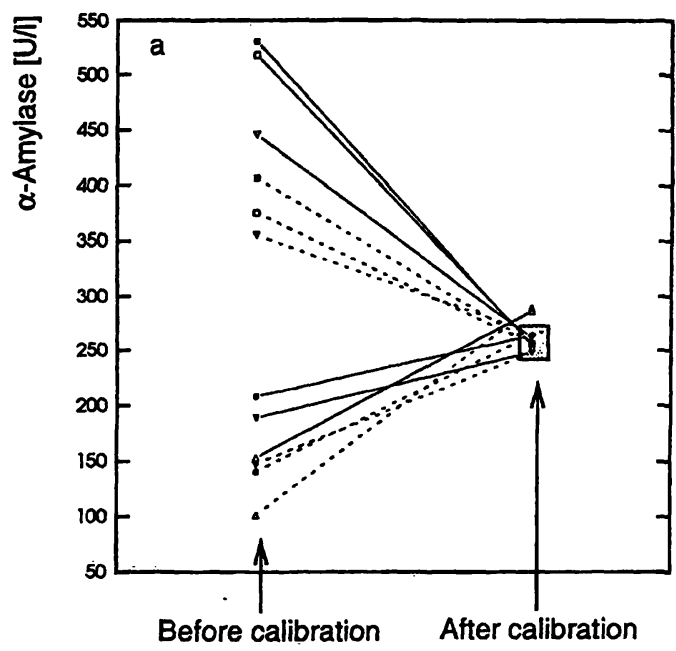

Fig. 1 Effect of calibration on the between-method coherency for $\alpha$-amylase activity. Each group corresponds to data obtained in one laboratory (a: lab. 1 ; b: lab. 2) at $30^{\circ} \mathrm{C}(--)$ and $37^{\circ} \mathrm{C}(-)$. Each sign corresponds to the mean enzyme catalytic concentration of patient samples for six measurement procedures using different

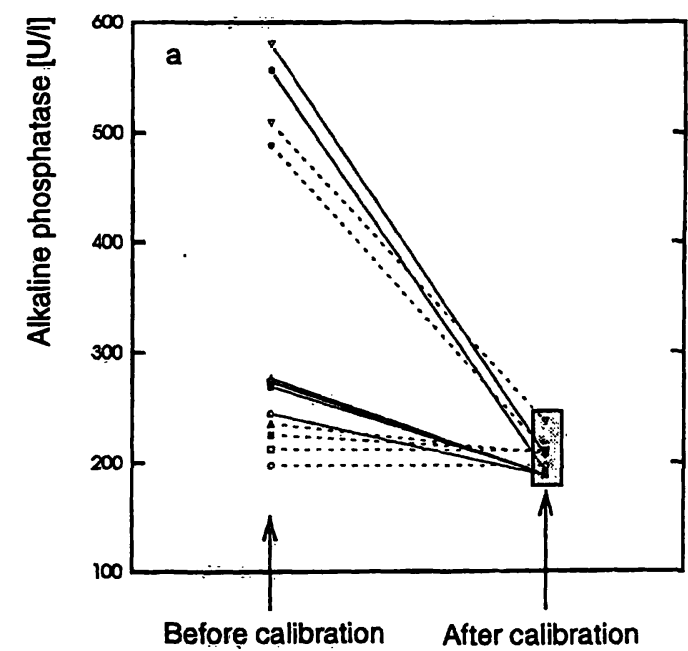

Fig. 2 Effect of calibration on the between-method coherency for alkaline phosphatase activity. Each graph corresponds to data obtained in one laboratory (a: lab. 2 ; b: lab 3) at $30^{\circ} \mathrm{C}(--)$ and $37^{\circ} \mathrm{C}$ (-). Each sign corresponds to the mean enzyme catalytic concentration of patient samples for eight measurement procedures, using as transphosphorylating buffer: 2-amino-2-methyl-1-propanol ac-
2 summarizes the calibration effect on the intermethod agreement. To quantify this effect, coefficients of variation were computerized for each series of mean values. A striking improvement was found for each enzyme and in each laboratory. After calibration, and after excluding maltotetraose results for $\alpha$-amylase and those obtained with a non-carboxylated substrate for $\gamma$-glutamyltransferase, all variation coefficients were less than $7.2 \%$. Maximal relative variations linked to the measurement procedures were also considerably decreased by calibration (tab. 2).

The temperature effect, i. e. the ratio of activity concentration at $37^{\circ} \mathrm{C}$ to that at $30^{\circ} \mathrm{C}$ for the different reagents

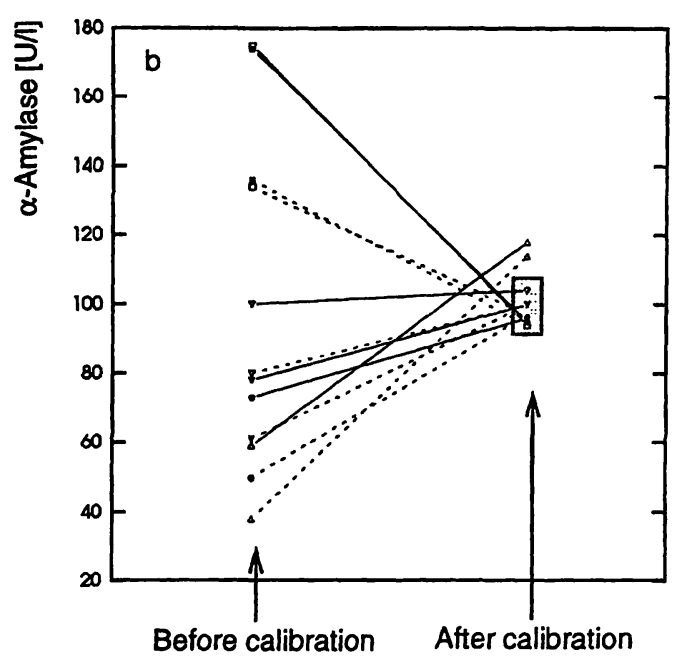

substrates: $\Delta$ maltotetraose (bioMérieux); 0 4-nitrophenylmaltoheptaoside (Boehringer); $\square$ ethylidene-4-nitrophenylmaltoheptaoside (Boehringer); $\oplus$ benzylidene-4-nitrophenylmaltoheptaoside (bioMérieux); $\nabla$ 2-chloro-4-nitrophenylmaltoheptaoside (Merck); $\nabla$ 2-chloro-4-nitrophenyl-maltotrioside (bioMérieux).

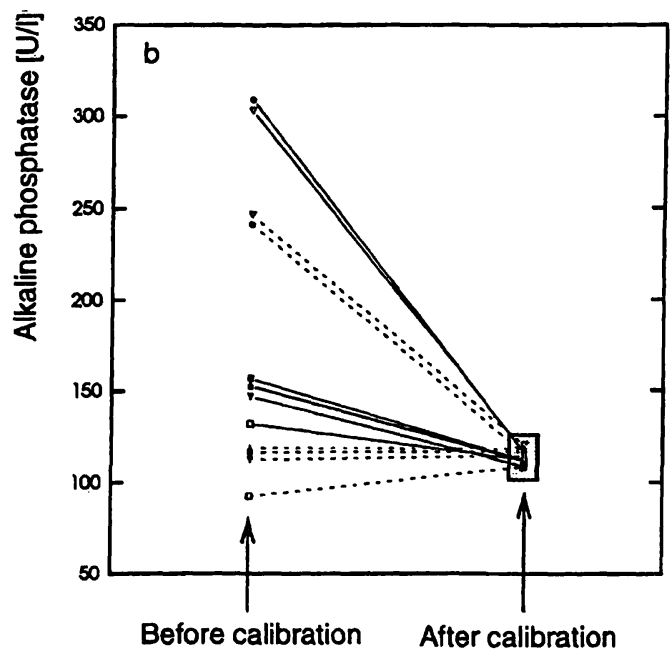

cording to IFCC recommendations ( $O$ home made reagent; $\triangle$ Roche), 2-amino-2-methyl-1-propanol according to SFBC recommendations ( $\square$ Boehringer; $\nabla$ bioMérieux; $\square$ Biotrol; $\triangle$ Roche) and diethanolamine according to DGKC recommendations ( $\nabla$ Boehringer; $\bullet$ bioMérieux). 


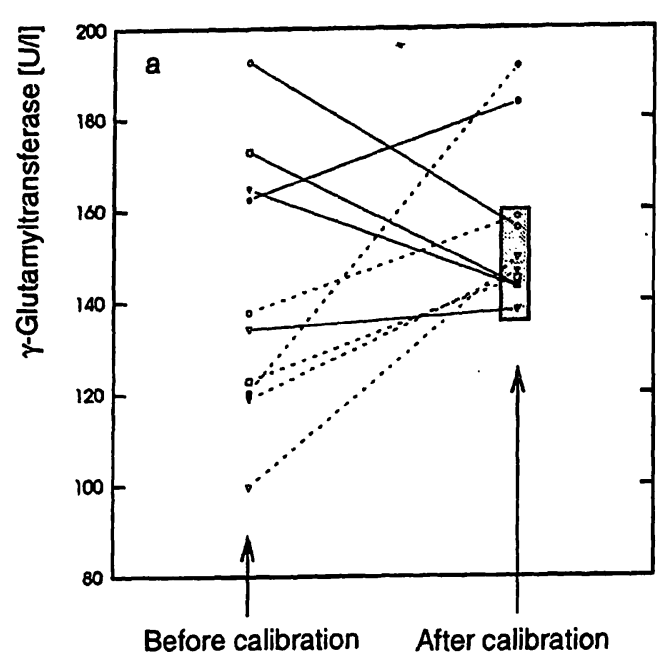

Fig. 3 Effect of calibration on the between-method coherency for $\gamma$-glutamyltransferase. Each graph corresponds to data obtained in one laboratory (a: lab. 4 ; b: lab. 5) at $30^{\circ} \mathrm{C}(--)$ and $37^{\circ} \mathrm{C}(-)$. Each sign corresponds to the mean enzyme catalytic concentration of patient samples for five measurement procedures using different

in two laboratories, is depicted in figure 4. Before calibration, it differed considerably with the used technique, especially for $\alpha$-amylase and alkaline phosphatase. The mean values and standard deviations calculated before and after calibration were respectively $1.37 \pm 0.11$ and $1.00 \pm 0.03$ for $\alpha$-amylase, $1.28 \pm 0.08$ and $0.94 \pm 0.03$ in the case of alkaline phosphatase and $1.34 \pm 0.05$ and $0.97 \pm 0.03$ for $\gamma$-glutamyltransferase. These results indicate that the differences between the temperature effects of techniques are reduced by calibration. Furthermore, the mean values of the ratios were close to 1.0 for the three enzymes after calibration.

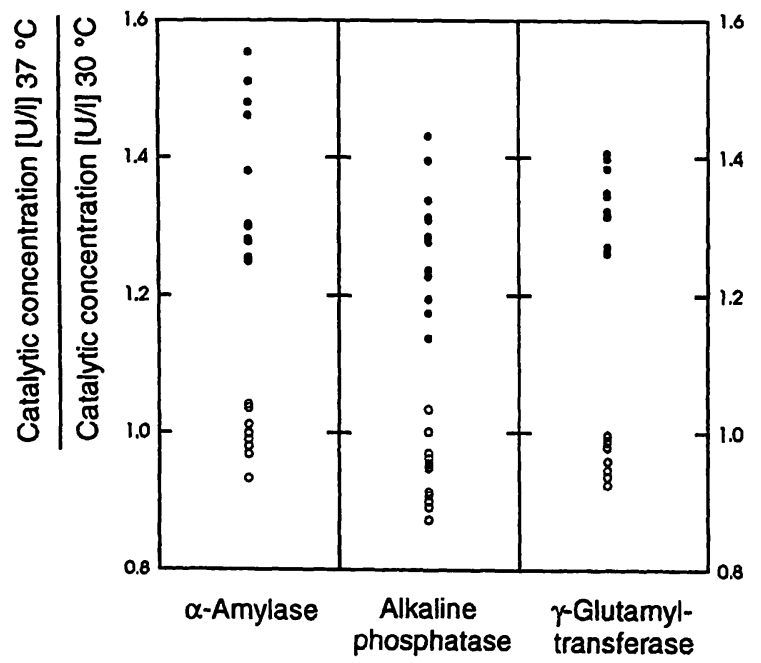

Fig. 4 Effect of reaction temperature on the activity concentration according to the used method of measurement. Each circle corresponds to a ratio for one procedure in one laboratory ( $\bullet$ before calibration; $O$ after calibration).

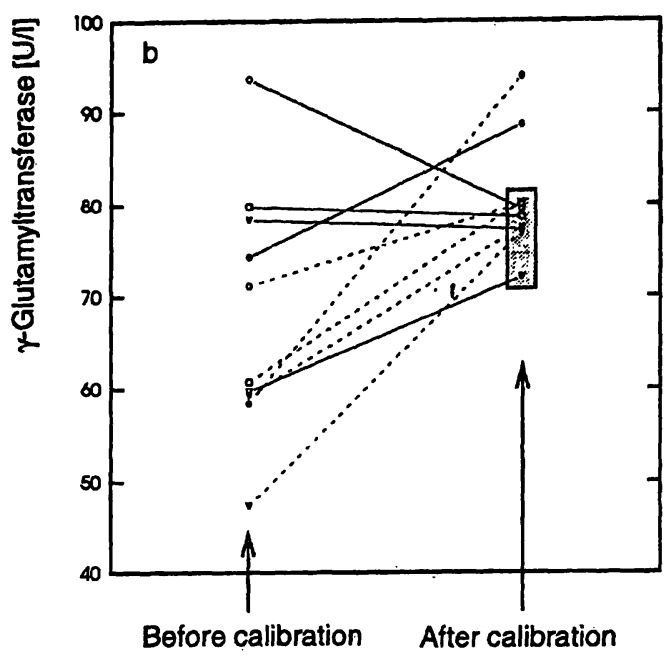

substrates at different concentrations. $\gamma$ - $L$-glutamyl-3-carboxy-4nitroanilide (O IFCC method with a home made reagent; $\nabla$ Boehringer; $\square$ Merck) and $\gamma$ - $L$-glutamyl-4-nitroanilide ( $\odot$ BioMérieux; $\nabla$ Biotrol).

\section{Discussion}

Three enzyme catalytic activity concentrations ( $\alpha$-amylase, alkaline phosphatase, $\gamma$-glutamyltransferase) were chosen for testing the effect of calibration because marked intermethod discrepancies were observed in external quality control assessments. Measurement procedures were selected according to their current use in Europe. CRM from BCR were employed as calibrators in this study because previous studies have demonstrated that these materials can be purified and stabilized, without significant alteration of their catalytic properties (3-8). Furthermore, CRM were shown to exhibit the same catalytic properties as the corresponding enzyme in patient samples. Another report indicated that the calibration approach with CRM 319 allowed the transfer of accuracy from the $\gamma$-glutamyltransferase reference method to another method which differs e.g. in its reaction temperature (7).

The variety of routine methods in use in clinical laboratories causes problems in the interpretation and comparison of results. $\alpha$-Amylase and alkaline phosphatase results may vary by a factor of up to 3.5 according to the measurement procedure. This factor is close to 2 for $\gamma$-glutamyltransferase. Measurement temperature is one of the numerous factors with modify the results of enzyme activity measurements. However, our data obtained with a large number of patient samples covering a wide range of catalytic activity concentration indicate that the temperature effect on enzyme activities varies not only with the enzyme in question, but also with the measurement procedure (kit of reagents, apparatus and application on the analyser). This indicates that a con- 


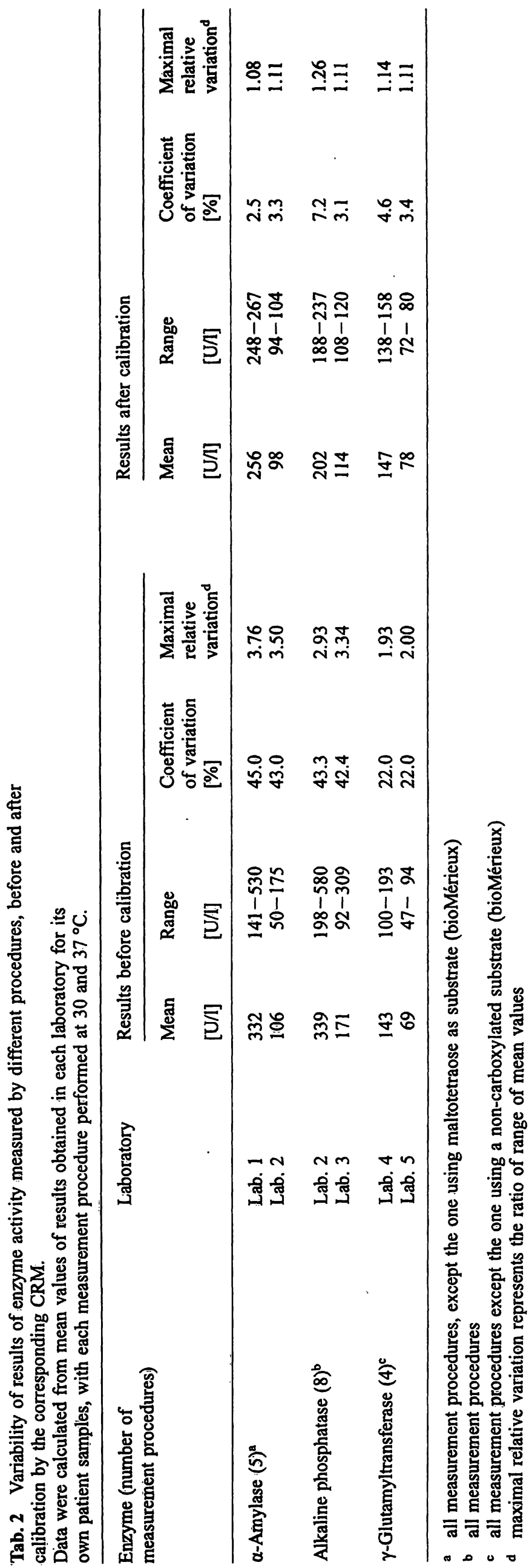

stant temperature factor cannot be established for an enzyme. After calibration, the effect of reaction temperature was not apparent under our conditions. This is explained by the fact that temperature ratios were found to be very similar for patient samples and CRM for each measurement procedure (data not shown).

The results obtained in this study indicate that the calibration approach with an appropriate material (i.e. CRM) may be applied to the three tested enzymes with most of the reagents used under routine conditions. Thus, these methods constitute a set of measurement procedures in which numeric coherency of results between methods is strongly improved after calibration. For alkaline phosphatase, the seven kits of reagents and the IFCC procedure with home made reagent gave very similar results after calibration in both laboratories. Among the six kits chosen for $\alpha$-amylase, five appeared as a set of procedures in the two laboratories. After calibration, the kit using maltotetraose gave different results, as compared with the homogeneous group. In fact, it is well known that salivary and pancreatic isoforms of amylase do not transform maltotetraose at the same rate (9). The feasibility of calibration has already been reported by Gerhardt et al. with kits including blue starch polymer, amylose, blocked starch, oligosaccharides and 4-nitrophenyloligosaccharides, with the same observation concerning maltotetraose $(10,11)$. In other words, the maltotetraose procedure did not show the same analytical specificty as other procedures using a 4-nitrophenyloligosaccharide as substrate. In the case of $\gamma$-glutamyltransferase (five reagents), the discrepancy of a noncarboxylated substrate was also observed in both participant laboratories. It indicates that not only the reaction principle has to be taken into account when selecting a measurement procedure. Surprisingly, $\gamma$-glutamyltransferase which is the sole enzyme without isoenzyme heterogeneity, showed the least favourable effect of calibration. This observation is in agreement with a previous one concerning commutability studies (7). These authors found that various kits showed higher significant differences when comparing CRM 319 and human sera than those observed with home made reagents according to international and national (British, French and Scandinavian) recommendations. Some differences, such as concentrations of substrate or effectors and/or additives, may affect CRM and patient samples in a different manner. As this effect is not predictable, it has to be checked experimentally using a large number of patient samples and the candidate material(s) as enzyme calibrator. It has to be stressed that an enzyme preparation cannot be used as a calibrator without prior validation. Lipase activity provides a good example of how the use of calibrators is not sufficient to correct inter-assay disagreement. This enzyme is most often determined routinely by tech- 
niques employing calibrators, ,but results may differ by a factor of up to 16 according to the measurement procedure (12). In fact, it has been shown that calibration with an appropriate material considerably improves inter-assay agreement (12). The importance of commutability of a material for intermethod calibration has also been recently discussed for amylase (11). The authors showed that materials with $\alpha$-amylase of non-human origin were not commutable with the enzyme in human sera and should not used for calibration.

\section{Conclusion}

It is possible to extensively reduce intermethod and interlaboratory discrepancies by the use of proper calibrators. Our data indicated that reaction temperature was

\section{References}

1. Tietz N, Rinker AD, Shaw LM. IFCC methods for the measurement of catalytic concentration of enzymes. Part 5: IFCC method for alkaline phosphatase (orthophosphoric-monoester phosphohydrolase, alkaline optimum EC 3.1.3.1.). J Clin Chem Clin Biochem 1983; 21:731-48.

2. Shaw LM, Strømme JH, London JL, Theodorsen L. IFCC methods for the measurement of catalytic concentration of enzymes. Part 4: IFCC method for $\gamma$-glutamyltransferase, $[\gamma$-glutamyl-peptide: amino acid $\gamma$-glutamyltransferase EC 2.3.2.2.]. J Clin Chem Clin Biochem 1983; 21:633-46.

3. Schiele F, Müller J, Colinet E, Siest G. Certification of an enzyme reference material for alkaline phosphatase (CRM371). Clin Biochem 1991; 24:159-68.

4. Schiele F, Müller J, Colinet E, Siest G. Production and certification of an enzyme reference material for $\gamma$-glutamyltransferase (CRM319). Part 1: Preparation and characterization. Clin Chem 1987; 33:1971-7.

5. Gella FJ, Gubern G, Canalias F. The certification of the catalytic concentration of human pancreatic $\alpha$-amylase in a reconstituted lyophilized material CRM 318. Commission of the European Communities, Community Bureau of Reference, Luxembourg. In press.

6. Schiele F, Siest G, Moss DW, Colinet E. The certification of the catalytic concentration of alkaline phosphatase in a reconstituted lyophilized material CRM 371. Commission of the European Communities, Community Bureau of Reference, Luxembourg, 1988.

7. Schiele F, Siest G, Moss DW, Colinet E. The certification of the catalytic concentration of gamma-glutamyltransferase in a reconstituted lyophilized material CRM 319. Commission of the European Communities, Community Bureau of Reference, Luxembourg, 1986. not a critical factor and that most of the tested reagents and procedures may be calibrated for the three enzymes tested. Nevertheless, at the present time, we have not yet found any commercial preparations that could be used as an enzyme multicalibrator. Secondary materials should be validated for each measurement procedure, using patient samples, prior their routine use as enzyme calibrators.

\section{Acknowledgements}

We wish to thank Prof. F. J. Gella (Barcelona) for providing the a-amylase preparation, Mrs $E$. Laurent (Strasbourg), A. Nicolas (Paris), M. Bondon and M. C. Deldon (Lyon) and M. P. Recouvreur and $S$. Bastien (Vandœuvre-lès-Nancy) for their technical assistance, and Société Française de Biologie Clinique for a financial support.

8. Moss DW, Maire I, Calam DH, Gaines Das RE, Lessinger JM, Gella FJ, Férard G. Reference materials in clinical enzymology: preparation requirements and practical interests. Ann Biol Clin 1994; 52:189-98.

9. Saito N, Horiuchi T, Yoshida M, Imai T. Action of human pancreatic and salivary $\alpha$-amylase on maltooligosaccharides: evaluation of kinetic parameters. Clin Chim Acta 1979; 97:253-60.

10. Gerhardt W, Waldenstrøm J, Hörder M, Magid E, Strømme H, Theodorsen $\mathrm{L}$, et al. SCE Nordic $\alpha$-amylase method selection and calibration study. A report by the Committee of Enzymes of the Scandinavian Society for Clinical Chemistry (SCE). Scand J Lab Invest 1985; 45:397-404.

11. Gerhardt W, Waldenstrøm J, Hörder M, Magid E, Strømme $H$, Theodorsen L, et al. SCE Nordic $\alpha$-amylase study II: assessment of proposed calibration procedure. A report by the Scandinavian Committee on Enzymes (SCE). Scand J Lab Invest 1986; 46:465-9.

12. Lessinger $\mathrm{JM}$, Férard $\mathrm{G}$. Plasma pancreatic lipase activity: from analytical specificity to clinical efficiency for the diagnosis of acute pancreatitis. Eur J Clin Chem Clin Biochem 1994; $32: 377-81$.

13. Gruben G, Canalias F, Gella FJ. Determination of $\alpha$-amylase activity: methods comparison and commutability study of several control materials. Clin Chem 1995; 41:435-8.

Prof. G. Férard

Laboratoire

Centre de Traumatologie et d'Orthopédie

10 , avenue Baumann BP96

F-67403 Illkirch-Graffenstaden Cedex

France 